\title{
Bacterial Foraging Optimized Hybrid Fuzzy Precompensated PD Control of Two Link Rigid-Flexible Manipulator
}

\author{
Srinivasan Alavandar \\ Department of Electronics and Computer Engineering, Caledonian College of Engineering, \\ Glasgow Caledonian Univeristy, PC 111 Seeb, Oman \\ E-mail: seenu.phd@gmail.com \\ Tushar Jain \\ Department of Electronics and Computer Engineering, Indian Institute of Technology Roorkee, \\ Roorkee, Uttarkhand 247667, India \\ E-mail:tushaar.jain@gmail.com \\ M J Nigam \\ Department of Electronics and Computer Engineering, Indian Institute of Technology Roorkee, \\ Roorkee, Uttarkhand 247667, India \\ E-mail: mkndnfec@,iitr.ernet.in \\ Received: 10-11-2008 \\ Revised: 14-03-2009
}

\begin{abstract}
Light-weight flexible arms will most likely constitute the next generation robots due to their large payload carrying capacities at high speeds and less power demand. Control problem of robots with flexible members is more complex compared to rigid robots due to vibrations during the motion. This paper presents the social foraging behavior of Escherichia coli bacteria to optimize hybrid Fuzzy Precompensated Proportional - Derivative (PD) controller in trajectory control of two link rigid-flexible manipulator. Numerical simulation using the dynamic model of the two link rigid-flexible manipulator shows the effectiveness of the approach in trajectory tracking problems and the use of fuzzy precompensation has superior performance in terms of improvement in transient and steady state response, robustness to variations in loading conditions and ease to use in practice. Comparative evaluation with respect to genetic algorithm based optimization is presented to validate the controller design. The results presented emphasize that a satisfactory tracking precision could be achieved using hybrid Fuzzy Precompensated PD controller with bacterial foraging based optimization.
\end{abstract}

Keywords: Bacterial Foraging, Fuzzy logic, rigid-flexible manipulators, optimization, PD control.

\section{Introduction}

The increased requirement for high performance robotic systems quantifies by a high speed of operation, high end position accuracy, and lower energy consumption has triggered vigorous research thrust in various multidisciplinary areas such as design and control of light weight flexible robot arms. ${ }^{1}$ Their potential advantages over rigid ones ${ }^{2}$ include less mass, faster operation, lower energy consumption, higher loadcarrying capability, wider operation range and so on. On the contrary, structural flexibility also gives rise to elastic deflections, distributed parameters, nonlinearity, strong coupling, non minimum phase etc. Therefore, improving the control performance under disturbances becomes a major issue. The control aim of motion control for flexible manipulator is to ensure the end position track the given trajectory accurately under the action of control torque of each joint.

Many control methods have been proposed to control the flexible manipulators employing PD control, ${ }^{3}$ adaptive control, ${ }^{4,5}$ computed torque method, ${ }^{6}$ robust control, ${ }^{7}$ sliding mode control ${ }^{21}$ etc.

Fuzzy logic controllers (FLCs) offer an attractive alternative to conventional model-based control schemes. An FLC is basically a model-free control 
paradigm, where the control signal is calculated by fuzzy inference rather than from the system dynamics. This property makes an FLC suitable for controlling nonlinear, uncertain or ill-understood dynamic systems such as flexible manipulator systems. It has also been proved that an FLC works well in situations where there is unknown variation in plant parameters and structures. ${ }^{8,9}$

For rigid-flexible systems the equations of motion can be described by hybrid ordinary partial differential equations. In Ref.10, the modes of vibration and the deflections of the beam, due to a force at the end, are calculated and a model of the complete arm is proposed. A. S. Yigit ${ }^{3}$ also considered a rigid-flexible robot arm. In his work, the flexible and the rigid parts were separated by use of an elbow and the aim was to analyze the stability of PD control for the problem. The work supports the full equations of motion of the manipulator, but concludes that an analytical solution cannot be determined. Simulations were done with approximate results using Galerkin's method. Just as was the case in Ref 11, Ref.3 was discovered such that the PD controller had a significant overshoot and the elastic link of the actuator had a significant damped oscillation. De Luca and Siciliano ${ }^{12}$ present an extension of the result to a multi-link flexible manipulator with nonlinear dynamics.

Green and Sasiadek ${ }^{13,14}$ have studied tracking of a square trajectory by a two-link robot manipulator using inverse dynamics, optimal and fuzzy logic system control schemes based on rigid-link dynamics and a control law derived from proportional and derivative gains computed using the dominant vibration mode. Peng et $\mathrm{al}^{15}$ proposed fuzzy composed controller with a nonlinear compensatory and a PD controller by considering both the static and dynamic control properties of the system.

Development of bio-inspired swarm intelligence methodologies based on bacterial colony behavior is an emergent research area with similar population and evolution characteristics to those of genetic algorithms. $^{18,19}$ However; it differentiates in emphasizing the cooperative behavior among group members. Swarm intelligence ${ }^{16}$ is used to solve optimization and cooperative problems among intelligent agents, mainly in artificial network training, multiobjective optimization problems, cooperative and/or decentralized control, etc. Basically, chemotaxis is a foraging behavior that implements a type of optimization where bacteria try to climb up the nutrient concentration, avoiding noxious substances and search for ways of neutral media. Based on these biological concepts, the definition of an optimization model of Escherichia coli bacterial foraging ${ }^{17}$ is possible.

The requirement of controllers with faster response and higher accuracy introduces a challenge that the researchers have faced in different ways. In this paper, an algorithm based on the foraging behavior of E-coli bacteria, found in the human intestine, is used to optimize the coefficients of the Fuzzy precompensation and PD controller for the trajectory control of two link rigid-flexible manipulator is proposed. Fuzzy precompensated PD controller mainly concentrates in improving the performance of the PD controllers when applied to flexible manipulator control problems in terms of robustness to variations in loading conditions, improvement in transient as well as steady-state performance, and ease to use in practice. Comparative evaluation with respect to genetic algorithm based optimization is presented to validate the controller design. The organization of the paper is as follows; Section 1 begins with the brief introduction of the problem and literature survey that was carried out. Section 2 describes the dynamics of two link rigid flexible manipulator dynamics. In Section 3, the design of hybrid fuzzy precompensated controller is carried out.. Section 4 describes the use of bacterial foraging for the proposed controller. Section 5 follows the simulation and results of the proposed approach. Section 6 concludes the findings and section 7 lists the references.

\section{Two Link Rigid - Flexible Manipulator Dynamics}

The Flexible manipulator used for simulation purpose is shown in Figure 1. The arm used for simulation is a direct drive planar chain with two revolute joints and two links, the second of which the forearm is very flexible. A nonlinear dynamic model of the two link rigid flexible arm has been derived following a Lagrangian approach. Small deformations are assumed for the forearm, leading to a linear dynamics of the flexible part, so that the main nonlinearities in the model arise from the rigid body interactions between the two links. To compute the low frequency modes, the forearm link is considered as an Euler - Bernoulli beam 
of length $l_{2}$ and length of the rigid link as $l_{1}$, uniform density $\rho$, and constant elastic properties EI. With reference to Figure 1, for a link point $x \in\left[0, l_{2}\right], w(x, t)$ is the bending deflection measured from the axis passing through the center of mass of the forearm. Accordingly, $\theta_{2}$ is the angle between this same axis and the first rigid links and $\theta_{I}$ is the angle of first rigid link with $\mathrm{X}$ coordinate as shown in Figure 1.

Considering the slewing nature of the forearm, deformation, eigen functions have been obtained in Ref. 20. The second joint moment of inertia $J_{02}$ and the payload mass $M_{P}$ and the moment of inertia $J_{P}$ are explicitly included in the boundary conditions associated to the partial differential equation for $w(x, t)$.

An approximation of order $n$ of the deflection can be expressed as,

$w(x, t)=\sum_{i=1}^{n} \phi_{i}(x) \delta_{i}(t)$

with the time varying coordinates associated to the mode shapes,

$\phi_{i}(x)=C_{1, i} \sin \left(\beta_{i} x\right)+C_{2, i} \cos \left(\beta_{i} x\right)+C_{3, i} \sinh \left(\beta_{i} x\right)+C_{4, i} \cosh \left(\beta_{i} x\right)$

The coefficients C's are determined, up to a scaling factor which is chosen through normalization, from the imposed boundary conditions. The values $\beta_{i}$ are numerically obtained as the first $n$ roots of the characteristic equation. ${ }^{20}$

$c \cdot s h-s \cdot c h-\frac{2 M_{p}}{\rho} \beta_{i} s \cdot s h-\frac{2 J_{p}}{\rho} \beta_{i}^{3} c \cdot c h-\frac{J_{02}}{\rho} \beta_{i}^{3}(1+c \cdot c h)$

$-\frac{M_{p}}{\rho^{2}} \beta_{i}^{4}\left(J_{02}+J_{p}\right)(c \cdot s h-s \cdot c h)+\frac{J_{02} J_{p}}{\rho^{2}} \beta_{i}^{6}(c \cdot s h+s \cdot c h)$

$-\frac{J_{02} J_{p} M_{p}}{\rho^{3}} \beta_{i}^{7}(1-c \cdot c h)=0$

where $s=\sin \left(\beta_{i} l_{2}\right), c=\cos \left(\beta_{i} l_{2}\right), s h=\sinh \left(\beta_{i} l_{2}\right)$, and $c h=\cosh \left(\beta_{i} l_{2}\right)$. The natural angular frequencies $w_{i}$ of the flexible link are related to $\beta_{i}$ through $\beta_{i}^{4}=\rho w_{i}^{2} / E I$. Starting from the analysis, the lagrangian dynamics of the two link robot is derived in the standard way as,

$B(q) \ddot{q}+c(q, \dot{q})+K q+D \dot{q}=G u$

where $q=\left(\theta_{1}, \theta_{2}, \delta_{1}, \ldots \ldots \delta_{n}\right) \in R^{n+2}$, and with positive definite symmetric inertia matrix $B$, coriolis and centripetal terms $c$, and elasticity matrix $K$. Joint viscous

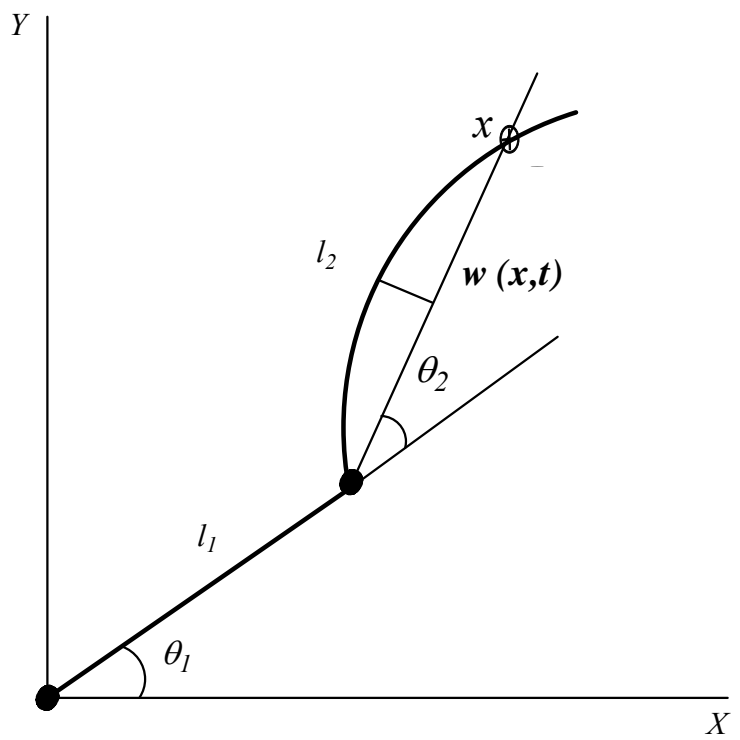

Fig. 1. Schematic diagram of rigid-flexible manipulator and its variables.

friction and modal damping coefficients are arranged on the diagonal of $D$, while input matrix $G$, transforms motor torques $u$ into generalized forces performing work on $q$. To express the single dynamic terms in (4), the following notation will be used;

$v_{i}=\rho \int_{0}^{l_{2}} \phi_{i}(x) d x, i=1, \ldots \ldots . .$,

and,

$\phi_{i e}=\left.\phi_{i}(x)\right|_{x=l_{2}}, \quad \phi_{i 0}^{\prime}=\left.\frac{\partial \phi_{i}(x)}{\partial(x)}\right|_{x=0}, \quad i=1, \ldots, n$

Since the eigen functions $\phi_{i}(x)$ automatically satisfy proper orthonormality conditions, relevant simplifications arise in the dynamic model. For control design purposes, we will consider only two modes of deformation, so that $q=\left(\theta_{1}, \theta_{2}, \delta_{1}, \delta_{2}\right) \in R^{4}$. Neglecting the Kinetic energy of the system which is quadratic or higher order in the deformation variables $\delta_{i}$ yields the inertia matrix,

$B(q)=\left[\begin{array}{cccc}b_{11} & b_{12} & b_{13} & b_{14} \\ b_{12} & b_{22} & 0 & 0 \\ b_{13} & 0 & 1 & 0 \\ b_{14} & 0 & 0 & 1\end{array}\right]$

with elements, 
$b_{11}=J_{1 T o t}+J_{1 T o t}+2 h_{3} \cos \theta_{2}-2\left(h_{1} \delta_{1}+h_{2} \delta_{2}\right) \sin \theta_{2}$

$b_{12}=J_{2 T o t}+h_{3} \cos \theta_{2}-\left(h_{1} \delta_{1}+h_{2} \delta_{2}\right) \sin \theta_{2}$

$b_{13}=h_{1} \cos \theta_{2}$

$b_{14}=h_{2} \cos \theta_{2}$

$b_{22}=J_{2 T o t}$

in which,

$h_{i}=\left(v_{i}+M_{P} \phi_{i e}\right) l_{2}, i=1,2$

$h_{3}=\left(M_{2} d_{2}+M_{P} l_{2}\right) l_{1}$

$J_{1 T o t}=J_{01}+J_{1}+M_{1} d_{1}^{2}+\left(M_{2}+M_{02}+M_{P}\right) l_{1}^{2}$

$J_{2 T o t}=J_{02}+J_{2}+M_{2} d_{2}^{2}+J_{P}+M_{P} l_{2}^{2}$

where, in addition to previous definitions, $l_{i}$ is the length of link $i, M_{i}$ and $M_{0 i}$ are the mass of the link $i$ and of joint $i, J_{i}$ and $J_{0 i}$ are their moments of inertia referred to the respective center of mass, and $d_{i}$ is the distance of the center of mass of link $i$ from joint axis $i$. The components of the coriolis and centripetal force vector $c(q, \dot{q})$ are,

$c_{1}=-\left(2 \dot{\theta}_{1} \dot{\theta}_{2}+\dot{\theta}_{2}^{2}\right)+\left[h_{3} \sin \theta_{2}-\left(h_{1} \delta_{1}+h_{2} \delta_{2}\right) \cos \theta_{2}\right]$

$\left.-2\left(\dot{\theta}_{1}+\dot{\theta}_{2}\right)\left(h_{1} \dot{\delta}_{1}+h_{2} \dot{\delta}_{2}\right) \sin \theta_{2}\right]$

$c_{2}=\dot{\theta}_{1}^{2}\left[h_{3} \sin \theta_{2}+\left(h_{1} \delta_{1}+h_{2} \delta_{2}\right) \cos \theta_{2}\right]$

$c_{3}=\dot{\theta}_{1}^{2} h_{1} \sin \theta_{2}$

$c_{4}=\dot{\theta}_{1}^{2} h_{2} \sin \theta_{2}$

The input matrix takes the form,

$G=\left[\begin{array}{c}I \\ G_{\delta}\end{array}\right], G_{\delta}=\left[\begin{array}{cc}0 & \phi_{10}^{\prime} \\ 0 & \phi_{20}^{\prime}\end{array}\right]$

while the elasticity matrix becomes,

$K=\left[\begin{array}{cc}0 & 0 \\ 0 & K_{\delta}\end{array}\right], K_{\delta}=\left[\begin{array}{cc}\omega_{1}^{2} & 0 \\ 0 & \omega_{2}^{2}\end{array}\right]$

Also, modal damping is included by specifying,

$D=\left[\begin{array}{cc}0 & 0 \\ 0 & D_{\delta}\end{array}\right], D_{\delta}=\left[\begin{array}{cc}2 \zeta_{1} \omega_{1} & 0 \\ 0 & 2 \zeta_{2} \omega_{2}\end{array}\right]$

where the first zeros on the diagonal of $D$ are due to the fact that the low friction at the joints is neglected. The above explicit expressions can be generalized to the case of $n>2$ modes in a straightforward way. And the tip deflection of the forearm is written as, $y_{\text {tip }}=\left(\frac{\phi_{1 e}}{l_{2}}-\phi_{10}^{\prime}\right) \delta_{1}+\left(\frac{\phi_{2 e}}{l_{2}}-\phi_{20}^{\prime}\right) \delta_{2}$

\section{Hybrid Fuzzy Precompensated PD Controller Design}

Conventional control methods, such as PD and PID controllers, are widely used in industrial applications. Such controller suffers from the problem of large overshoots and undershoots in its output response when applied to systems containing distributed parameters, nonlinearity, strong coupling. In this work, a novel fuzzy logic-based precompensation approach for controlling system with high nonlinearity is attempted. The control structure consists of a fuzzy logic-based precompensator followed by a conventional PD controller.

\subsection{PID Control}

In this section we describe a general PD (ProportionalDerivative) controller for the control of rigid-flexible link arm. The control input to the $i^{\text {th }}$ actuator is given by,

$\tau_{i}=-K_{P i} \hat{e}_{i}(t)-K_{D i} \dot{\hat{e}}_{i}(t)$

where,

$\hat{e}_{i}(t)=\left[\hat{q}_{d i}(t)-q_{i}(t)\right]$

In Equation above, $\hat{e}_{i}(t)$ and $\dot{\hat{e}}_{i}(t)$ represent the error in the joint angle and velocity for the $i^{\text {th }}$ link. $K_{P i}$ and $\mathrm{K}_{\mathrm{Di}}$ are the proportional and derivative gains, respectively. Sufficient literature dealt PD control investigated that better response has not been achieved in case of this distributed parameters, nonlinearity, strong coupled systems.

\subsection{Fuzzy Precompensation}

The fuzzy precompensator uses the desired position $q_{d}$, and the plant output $q$, to generate a precompensated modified desired position $\hat{q}_{d i}(t)$. The fuzzy precompensator modifies the desired position, to compensate undershoot and overshoot in the output response, to eliminate the steady-state error and improve the performance of the output response for PD control systems with nonlinearities by introducing a fuzzy logic controller in front of the PD controller. Fuzzy precompensation that proposed is indeed insensitive to 
nonlinearities, and exhibits good transient and steadystate behavior.

A Fuzzy Precompensated PD controller was designed and applied to control the position of the manipulator, since it is well known that Fuzzy PD gives a faster transient than Fuzzy PI type. The fuzzy precompensator is described by the equations,

$e_{i}(t)=\left[q_{d i}(t)-q_{i}(t)\right]$

$\gamma(t)=F\left[e_{i}(t)-\dot{e}_{i}(t)\right]$

$\hat{q}_{d i}(t)=q_{d i}(t)+\gamma(t)$

The inputs to the precompensator are the desired position $q_{d i}(t)$ and the plant actual position $q_{i}(t)$. The term $F\left[e_{i}(t)-\dot{e}_{i}(t)\right]$ is a nonlinear mapping of $e_{i}(t)$ and $\dot{e}_{i}(t)$ based on fuzzy logic (described below). The term $\gamma(t)=F\left[e_{i}(t)-\dot{e}_{i}(t)\right]$ represents a compensation or correction term, so that the compensated/modified desired position $\hat{q}_{d i}(t)$ is simply the sum of the external desired position $q_{d i}(t)$ and $\gamma(t)$. The correction term is based on the error $e_{i}(t)$, and the change of error $\dot{e}_{i}(t)$.

The compensated command signal $\hat{q}_{d i}(t)$ is used as the input to the PD controller, as shown in Figure 2 in the overall block diagram of the hybrid fuzzy precompensated PD controller.

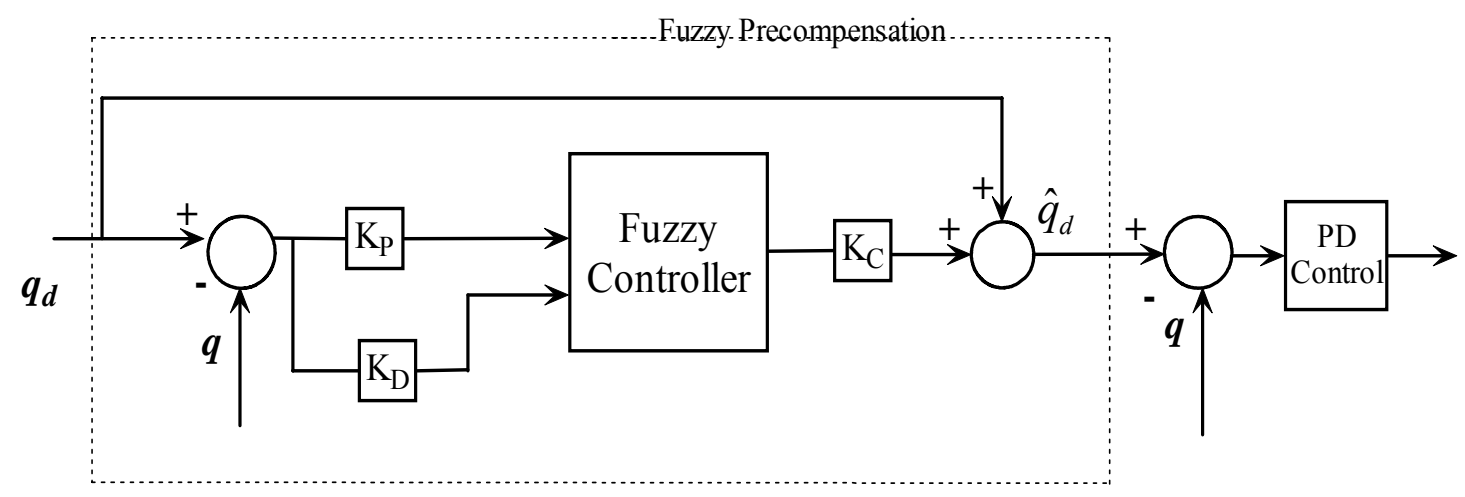

Fig. 2. Overall block diagram of the proposed hybrid controller.

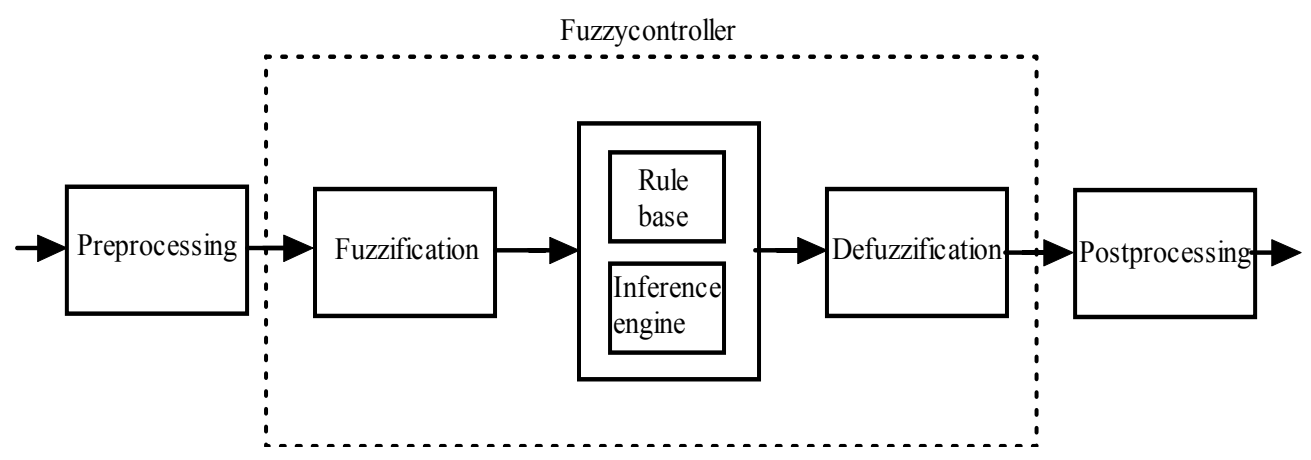

Fig. 3. Structure of Fuzzy controller

Figure 3 shows the structure of Fuzzy controller. It consists of a preprocessing, fuzzification interface, knowledge base, fuzzy inference system, defuzzification interface and a post processing unit. The preprocessing block transforms the input ( $e$ and $\dot{e}$ ) on the actual universe of discourse (UOD) to the normalized universe of discourse, using the input scaling factors $K_{P}, K_{D}$ and $K_{C}$ for computational simplicity.

The fuzzification block converts crisp inputs to appropriate fuzzy sets using the membership functions as shown in Figure 4. The knowledge base provides the membership functions and the linguistic control rules. 
The fuzzy inference engine performs fuzzy reasoning, based on the linguistic control rules, using Zadeh's compositional rule of inference. ${ }^{22}$ The defuzzification block generates a crisp control output $u(t)$ by utilizing the centre of gravity method,

$u(t)=\frac{\sum \mu_{i}(u) u_{i}}{\sum \mu_{i}(u)}$

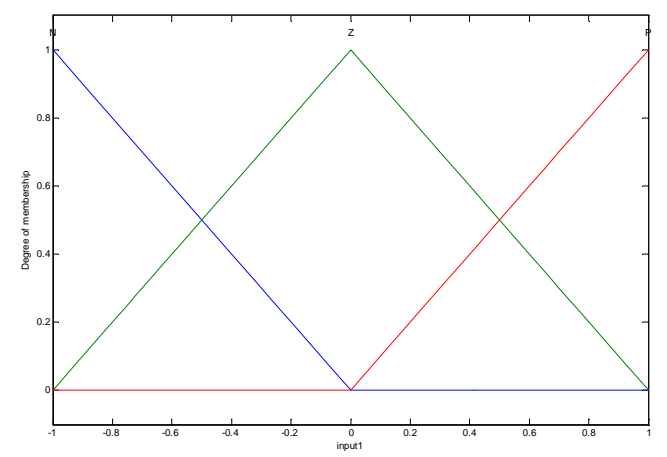

Fig. 4. Membership functions of input and output variables for Fuzzy Precompensated Controller

Table 1 shows the rules of Fuzzy precompensated PD control and Figure 5 represents the output surface of the fuzzy precompensated controller with the rule base.

Table 1 Fuzzy Precompensated PD Controller rules

\begin{tabular}{|c|c|c|c|c|}
\hline & \multicolumn{3}{|c|}{$\begin{array}{l}\text { Change of } \\
\text { Error (ed) }\end{array}$} \\
\hline & & $\mathrm{N}$ & $\mathrm{Z}$ & $\mathrm{P}$ \\
\hline \multirow{3}{*}{ 氠 } & $\mathrm{N}$ & $\mathrm{N}$ & $\mathrm{N}$ & $\mathrm{Z}$ \\
\hline & Z & $\mathrm{N}$ & $\mathrm{Z}$ & $\mathrm{P}$ \\
\hline & $\mathrm{P}$ & $\mathrm{Z}$ & $\mathrm{P}$ & $\mathrm{P}$ \\
\hline
\end{tabular}

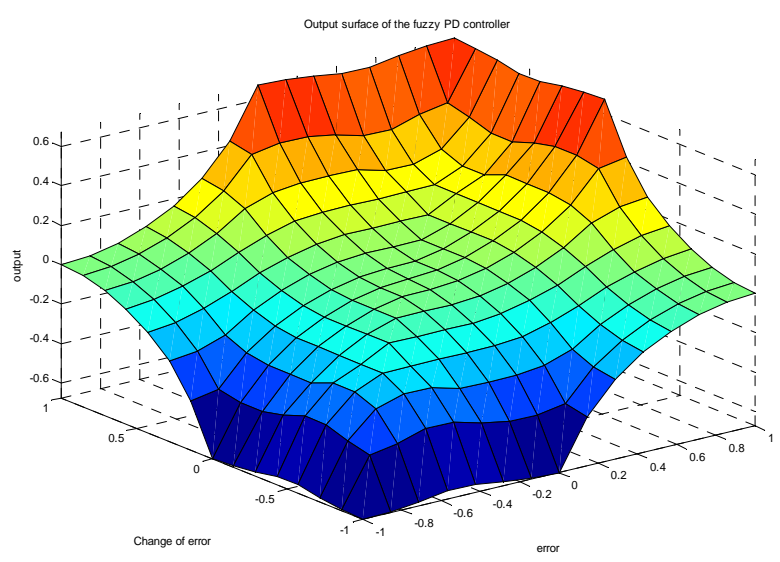

Figure 5. Output surface of the fuzzy PD controller

\section{Bacterial Foraging}

This paper considers the foraging behavior of E. coli, which is a common type of bacteria. ${ }^{17}$ Its behavior to move comes from a set of up to six rigid 100-200 rps spinning flagella, each driven as a biological motor. An E. coli bacterium alternates between running and tumbling. Running speed is $10-20 \mu \mathrm{m} / \mathrm{sec}$, but they cannot swim straight.

The main goal based on bacterial foraging is to apply in order to find the minimum of $J(\phi), \phi \in R^{n}$, not in the gradient $\nabla J(\phi)$. here, when $\phi$ is the position of a bacterium, and $J(\phi)$ is an attractant-repellant profile. That is, it means where nutrients and noxious substances are located, so $J(\phi)<0, J(\phi)=0, J(\phi)>0$ represent the presence of nutrients, a neutral medium and the presence of noxious substances, respectively can be shown by,

$$
P(j, k, l)=\left\{\phi^{i}(j, k, l) \mid i=1,2, \ldots ., N\right\}
$$

Eq. (19) represents the positions of each member in the population of the $\mathrm{N}$ bacteria at the $\mathrm{j}^{\text {th }}$ chemotactic step, $\mathrm{k}^{\text {th }}$ reproduction step, and $\mathrm{l}^{\text {th }}$ elimination-dispersal event.

Basically, chemotaxis is a foraging behavior that implements a type of optimization where bacteria tries to climb up the nutrient concentration (find lower and lower values of $J(\phi)$ ), avoid noxious substances, and search for ways out of neutral media. It implements a biased random walk. This paper describes the method in the form of an algorithm to search optimal values of Hybrid Fuzzy precompensated PD controller parameters discussed above. The overall block diagram of the same is shown in figure 6 .

In this paper, the bacterial foraging scheme has been used for the optimization of controller parameters shown in Figure 6. The integral square error (ISE) performance index is used as the objective function " $J$ " for optimization

$$
J=I S E=\int_{0}^{t} e^{2}(t) d t, \text { where } e(t)=q_{d}(t)-q(t)
$$

Here in our simulation for tuning the fuzzy precompensated PD contoller, dimension of search space is 7 with 10 numbers of bacteria, 4 number of chemotactic steps, length of the swim and number of reproduction steps as 4 , and 2 number of elimination dispersal events is considered. 


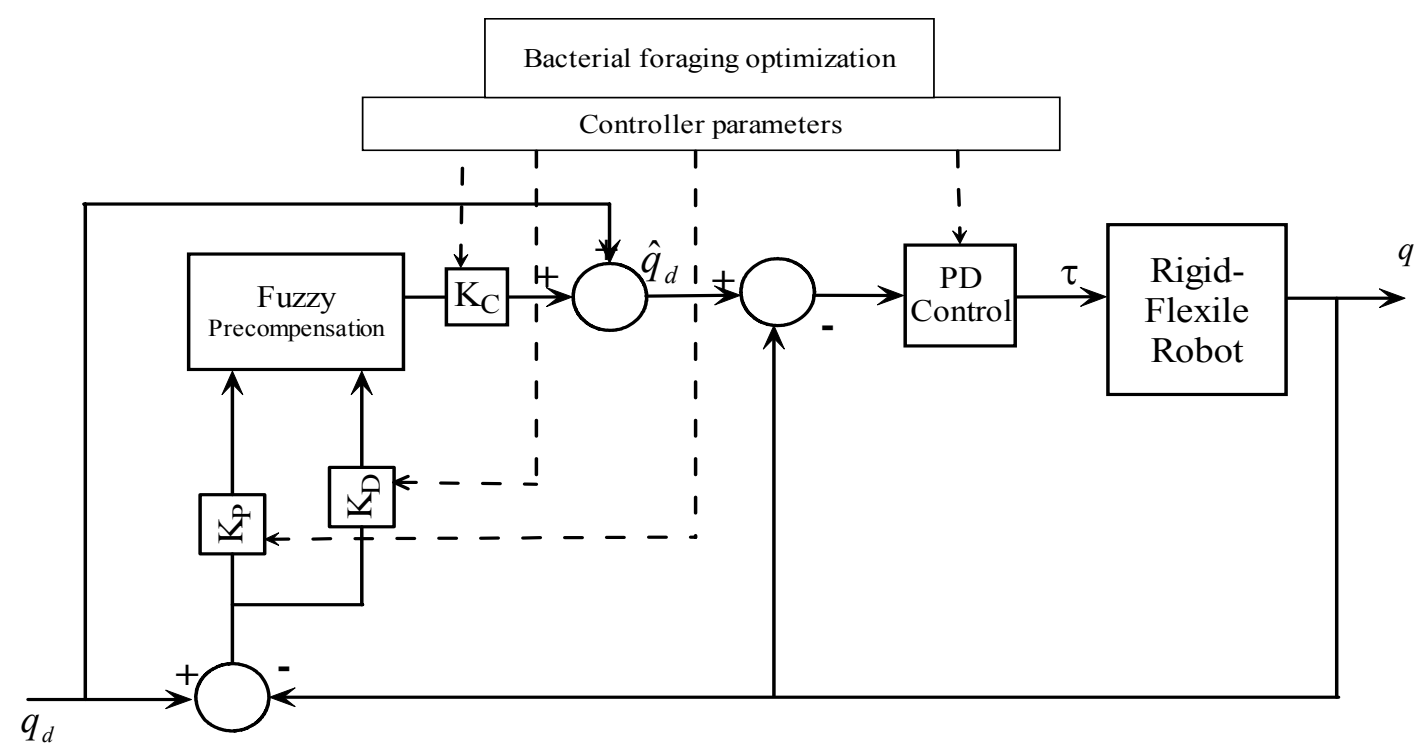

Fig. 6. Bacterial Foraging optimized Fuzzy Precompensated PD Control

\section{Simulation and Results}

In this section, the simulation was run under MATLAB 7.01 with Fuzzy Logic Toolbox 2.2 (R14SP1). To demonstrate the effectiveness of the proposed Fuzzy precompensated PD controller optimized by bacterial foraging algorithm, the dynamic model of two link rigid flexible arm is considered. The flexible robot arm considered is characterized by following data in Table 2 ;

Table 2. Simulation parameters

\begin{tabular}{|l|l|l|}
\hline$l_{1}=0.3 \mathrm{~m}$ & $h_{1}=0.336 \mathrm{~kg} \mathrm{~m}^{2}$ & $\begin{array}{l}\omega_{2}=14.395 .2 \pi \\
\mathrm{rad}_{\mathrm{sec}^{-1}}\end{array}$ \\
\hline$l_{2}=0.7 \mathrm{~m}$ & $h_{2}=0.126 \mathrm{~kg} \mathrm{~m}^{2}$ & $\zeta_{1}=0.07$ \\
\hline$J_{\text {ITot }}=0.447 \mathrm{~kg} \mathrm{~m}^{2}$ & $h_{3}=0.195 \mathrm{~kg} \mathrm{~m}^{2}$ & $\zeta_{2}=0.03$ \\
\hline$J_{\text {ITot }}=0.303 \mathrm{~kg} \mathrm{~m}^{2}$ & $\phi^{\prime} 10=5.74$ & $\phi_{1 e}=-1.446 \mathrm{~m}$ \\
\hline$J_{02}=6.35 \times 10^{-4} \mathrm{~kg} \mathrm{~m}^{2}$ & $\phi_{20}^{\prime}=11.64$ & $\phi_{2 e}=1.369 \mathrm{~m}$ \\
\hline$M_{P}=J_{P}=0$ & $\begin{array}{l}\omega_{1}=4.16 .2 \pi \\
\mathrm{rad} / \mathrm{sec}^{-1}\end{array}$ \\
\hline
\end{tabular}

Figure 7 shows the desired joint position profiles for the simulation to evaluate the effectiveness of the proposed approach. Figure 8 shows the modified or compensated desired position $q_{i}(t)$ for $i=1,2$ using fuzzy precompensation. Figure 9 shows the Joint position error profiles for the two joints using the proposed bacterial foraging optimized fuzzy precompensated PD controller (BFFPPDC). The same controller is compared by optimizing with genetic algorithm and is shown in figure 10. Table 3 constitutes the integral square errors for the two joints using the proposed bacterial foraging optimized fuzzy precompensated PD controller (BFFPPDC) and genetic algorithm optimized fuzzy precompensated PD controller (GAFPPDC). It is found from the simulation results that the tracking error is reduced to a great extent by applying our proposed approach. This is demonstrated by the calculation of integral square error for various joints as seen in Table 2 .

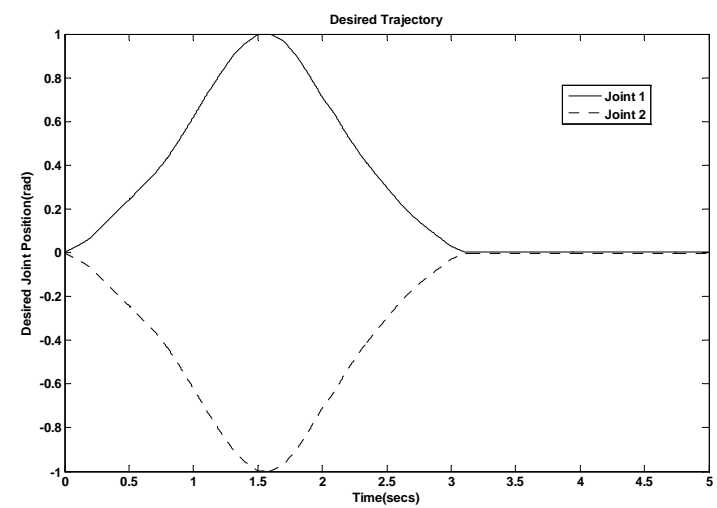

Fig. 7. Desired position profile 


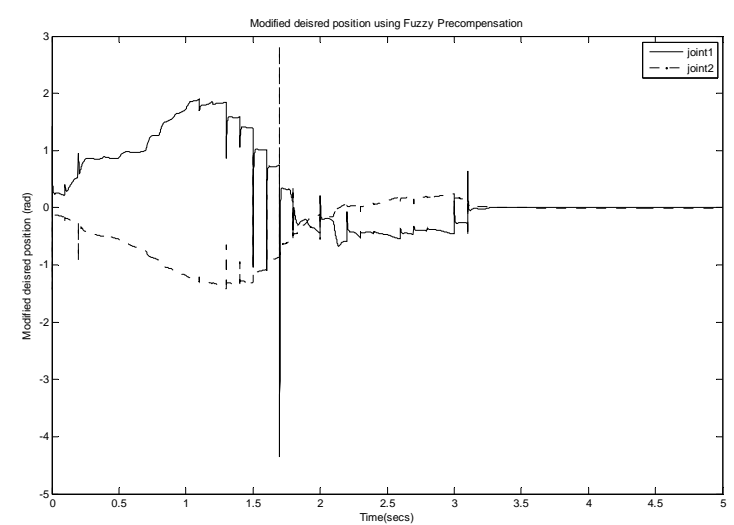

Fig.8. Modified desired position using fuzzy precompensation.

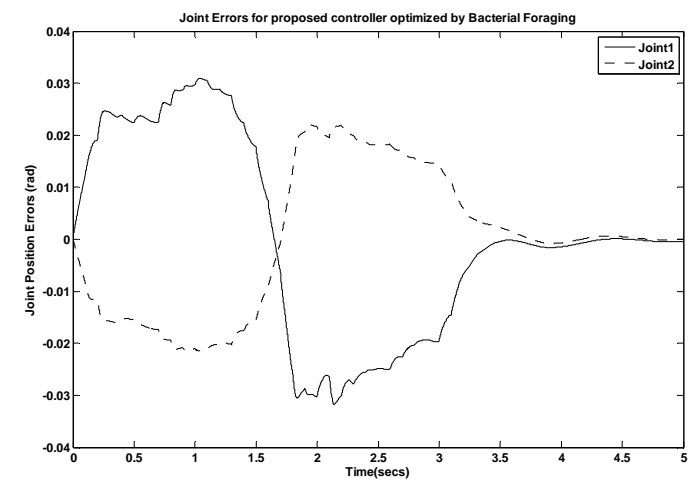

Fig. 9. Error profiles for the two joints using BFFPPDC.

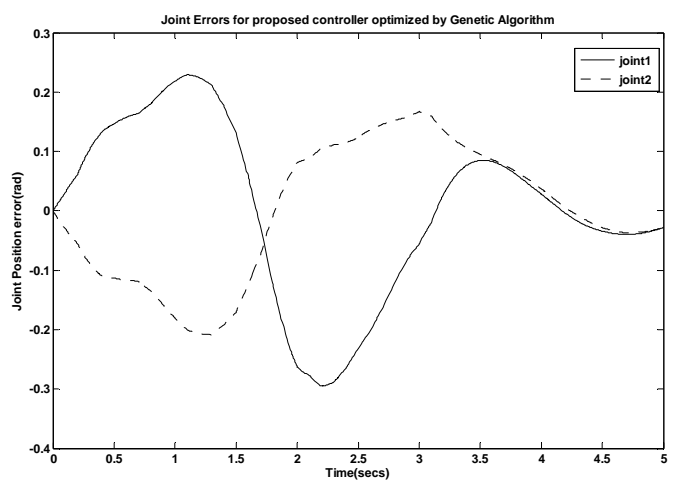

Fig. 10. Error profiles for the two joints using GAFPPDC.
Table 2. Integral square errors for various joints

\begin{tabular}{|l|l|l|}
\hline Controller & Joint1 & Joint2 \\
\hline GAFPPDC & 0.1059 & 0.0643 \\
\hline BFFPPDC & 0.0018 & 0.0009 \\
\hline
\end{tabular}

\section{Conclusions}

The bacterial foraging (BF) algorithm is used to optimize the gains of hybrid fuzzy precompensated PD controller for a two link rigid-flexible manipulator in trajectory tracking applications. Also, the proposed fuzzy logic based precompensation scheme consisting $f$ fuzzy PD precompensator and a conventional PD Controller has superior steady state and transient performance, good stabilization and tracking performance compared to a conventional PD controller.An advantage of our present approach is that an existing PD controller can be easily modified into our control structure by simply adding a fuzzy precompensator. The proposed bacterial foraging (BF) algorithm is compared with genetic algorithm (GA) and it is found that $\mathrm{BF}$ converges faster than GA and shows much superior performance in terms of joint errors and computational time. Optimization of fuzzy membership functions with bacterial foraging and comparison with other swarm intelligence based techniques will be of further study.

\section{References}

1. W. J. Book, Modeling, design, and control of flexible manipulator arms: A tutorial review. Proc. IEEE Conf. Decision and Control, pp. 500-506, 1990.

2. A. R. Fraser, and R.W. Daniel, Perturbation techniques for flexible manipulators. Massachusetts: Kluwer Academic Publishers; 1991.

3. A. S. Yigit, On the stability of PD control for a two-link rigid-flexible manipulator, ASME J.Dyn. Systems Meas. Control, 116, 208-215, 1994.

4. L. C. Lin, and S. L. Yeh, A composite adaptive control with flexible quantity feedback for flexible-link manipulators, J. Robotic Systems, 13(5), 289-302, 1996.

5. J. H. Yang, F. L. Lian, and L. C. Fu, Nonlinear adaptive control for flexible-link manipulators, IEEE Trans. Robotics Automat. 13(1), 140-148, 1997.

6. J. O. King, V. G. Gourishankar, and R. E. Rink, Composite pseudolink end-point control of flexible 
manipulators, IEEE Trans. Systems Man Cybernet., 20(5), 969-977, 1990.

7. Y. Morita, H. Ukai, and H. Kando, Robust Trajectory Tracking Control of Elastic Robot Manipulators, Trans. On ASME, J. Dvnamic Systems, Measurements, and Control, Vol.119, pp.727-735, Dec. 1997.

8. I. H. Suh, K. S. Eom, H. J. Yeo, and S. R. Oh, Fuzzy adaptive control of industrial robot manipulators with position servos. Mechatronics, 5(8), 899-918, 1995.

9. B. Subudhi, and A. S. Morris, Fuzzy and neuro-fuzzy approaches to control a flexible single-link manipulator, Proc. Instn Mech. Engrs Part I: J. Systems and Control Engineering, Vol. 217, 387-399, 2003.

10. F. L. Hu and A. G. Ulsoy, Dynamic Modelling of Constrained Flexible Robot Arms for Controller Design, ASME Journal of Dynamic Systems, Measurement and Control, Vol. 116, pp. 56-65, March 1994.

11. D. S. Kwon, and W. J. Book, A Time-Domain Inverse Dynamic Tracking Control of a Single-Link Flexible Manipulator, ASME Journal of Dynamic Systems, Measurement and Control, Vol. 116, pp. 193-200, June 1994.

12. A. De Luca, and G. Di Giovanni, Rest-to-Rest Motion of a Two-link Robot with a Flexible Forearm, Proceedings of IEEE/ASME International Conference on Advanced Intelligent Mechatronics, 929-935, July 2001.

13. A. Green, and J. Z. Sasiadek, Direct, optimal, stochastic and fuzzy control of a two link flexible manipulator. Proceedings of the AIAA Guidance, Navigation and Control Conference and Exhibit, Denver, CO, August 1417, pp.2000-4375, 2000.

14. A. Green, and J. Z. Sasiadek, Methods of trajectory tracking for flexible-link manipulators. Proceedings of the AIAA Guidance, Navigation and Control Conference and Exhibit, Monterey, CA, August 5-8, pp.2002-4565, 2002.

15. G. Peng, X. Wang, Y. Xue, Study on fuzzy PD control of planar two link flexible manipulator, Proceedings of IEEE TENCON'02, 1542-1545, 2002.

16. J. F. Kennedy, R. C. Eberhart, and Y. Shi, Swarm Intelligence, San Francisco: Morgan Kaufmann Pub, 2001.

17. K. M. Passino, Biomimicry of bacterial foraging for distributed optimization and control, IEEE Control Systems, vol. 22, no. 3, pp. 52-67, 2002.

18. M. N. H. Siddique, and M. O. Tokhi, GA-based Neural Fuzzy Control of Flexible-link Manipulators, Engineering Letters, Vol. 13, No. 2, pp. 148-157, 2006.

19. V. B. Nguyen, and A. S. Morris, Genetic Algorithm Tuned Fuzzy Logic Controller for a Robot Arm with Two-link Flexibility and Two-joint Elasticity, J Intell Robot Syst 49, pp. 3-18, 2007.

20. F. Bcllezza, L. Lanari, and G. Ulivi, Exact modeling of the slewing flexible link, Proc. of IEEE International Conference on Robotics and Automation, Cincinnati, $0 \mathrm{H}$, May 13 18, pp. 734-739, 1990.
21. I. Kalaykov, and B. Iliev, Time-optimal sliding mode Control of robot manipulators, in Proc. IEEE Conf. On Industrial Electronics, Control and Instrumentation IECON-2000, Nagoya, Japan, October 22-28, pp. 265270,2000

22. R. Fuller and H. J. Zimmermann, On Zadeh's compositional rule of inference, in: R. Lowen and $M$. Roubens eds., Proceedings of the Fourth IFSA Congress, Vol. Artifical Intelligence, Brussels, 1991 41-44. 